\title{
Impact of acquisition of 16S rRNA methylase RmtB on the fitness of
}

\section{Escherichia coli}

Bingming Ou ${ }^{a, b, 1}$, Lin Chen ${ }^{a, b, c, 1}$, Yujie Song ${ }^{a, b}$, Ying Yang ${ }^{a, b}$, Qian Zhang ${ }^{a, b}$, Yi Yang ${ }^{a, b}$, Luan Li ${ }^{a, b, d}$, Wai Liang Tham ${ }^{a, b, e}$, David H. Francis ${ }^{f}$, Guoqiang Zhu ${ }^{a, b, *}$

a College of Veterinary Medicine, Yangzhou University, Yangzhou 225009, China

b Jiangsu Co-Innovation Center for Important Animal Infectious Diseases and Zoonoses, Yangzhou 225009, China

c Jiangsu Agri-animal Husbandry Vocational College, Taizhou 225300, China

${ }^{\mathrm{d}}$ Faculty of Land and Food Systems, University of British Columbia, Vancouver, BC, V6T 1Z4, Canada

e Department of Biochemistry and Molecular Biology, University of British Columbia, BC, Vancouver V6T 1Z4, Canada

${ }^{\mathrm{f}}$ Department of Veterinary and Biomedical Sciences, South Dakota State University, Brookings, SD 57007, USA

Article history:

Received 28 January 2016

Accepted 23 February 2016

Keywords:

16S rRNA methylase RmtB

Deletion mutation

Aminoglycosides 
Growth rate

Competitive ability

Biofilm formation

* Corresponding author. Tel.: +86 5148797 2590; fax: +86 51487311374.

E-mail address: yzgqzhu@yzu.edu.cn (G. Zhu).

${ }^{1}$ These two authors contributed equally to this paper. 
The aim of this study was to elucidate the biological phenotypes of $16 \mathrm{~S}$ rRNA methylase RmtB in Escherichia coli and the impact of RmtB acquisition on the fitness of the target bacterium. An rmtB in-frame deletion mutant in E. coli was constructed using a suicide vector (pDMS197)-based double crossover allelic exchange, and its corresponding complemented strain was established. Combined studies of microdilution susceptibility testing, conjugation experiments, growth kinetics assays, competitive experiments, biofilm formation tests and motility assays were performed to study the $r m t B$-mediated fitness among the prototype $E$. coli strain, its isogenic mutant and the corresponding complemented strain. The minimum inhibitory concentrations (MICS) of 4,6-disubstituted 2-deoxystreptamines for the $r m t B$ wild-type strain, its isogenic mutant and the complemented strain were $\geq 1024$, $\leq 2$ and $\geq 1024 \mathrm{mg} / \mathrm{L}$, respectively. Both the growth rates and the competitive abilities of the wild-type and complemented strains were relatively inferior to the $\Delta r m t B$ mutant. There was no significant difference in biofilm formation and motility among the three strains. In conclusion, the data presented here suggest that acquisition of the $16 \mathrm{~S}$ rRNA methylase gene $r m t B$ in $E$. coli can exact a fitness cost on the bacteria, subsequently reducing the growth rate slightly and decreasing the competitive capacity of the bacterium, whereas it does not affect biofilm formation or motility. 


\section{Introduction}

Aminoglycosides are potent antibiotics active against aerobic Gram-negative bacteria and some Gram-positive bacteria. The mechanism of antibacterial activity involves binding to the highly conserved A site of $16 \mathrm{~S}$ rRNA in the $30 \mathrm{~S}$ small ribosomal subunit, leading to interference with the decoding of mRNA [1]. The 16S rRNA methyltransferase exists in antibiotic-producing bacteria and prevents the bacterium from being eliminated by its own secreted antibiotics; in the past this self-protective mechanism was once thought to be irrelevant in clinical settings [2]. However, a study by Yokoyama et al. [3] showed the existence of a drug resistance determinant, designated 16S rRNA methylase RmtA, in clinical settings. The unique resistance mechanism of these enzymes could involve blocking of aminoglycosides from binding to their target site by methylating one or several bases in the A site of the $16 \mathrm{~S}$ ribosomal subunit. This might involve using S-adenosylmethionine as the methyl donor, leading to a high level of resistance to aminoglycosides, thus resulting in the failure of antibiotic treatment. So far, ten kinds of $16 \mathrm{~S}$ rRNA methylases have been reported in Gram-negative bacteria from different countries and regions, including ArmA, RmtA, RmtB, RmtC [4], RmtD or RmtD 1 [5,6], RmtD 2 [5,6], RmtE [7], RmtF [8], RmtG [9], RmtH [10] and NpmA [11], which are encoded by at least 12 types of gene alleles.

It is known that antimicrobial resistance carries a fitness cost expressed in terms of reduced growth rate, competitive ability or virulence [12]. This cost of antimicrobial resistance is predicted to play a key role in the evolutionary dynamics of resistance, because the presence of resistance generates selection against resistance. However, some recent studies have found that the costs of aminoglycoside methylases are highly variable owing to a wide variety of factors, and thus estimating their potential distribution and evolutionary dynamics is difficult [13-16]. Recent epidemiological investigations have frequently reported the presence of 16S rRNA methylase RmtB in clinical and veterinary strains from various geographic areas, including East Asia, Europe and the Americas [4,17-20], notably increasingly involving bacterial strains isolated and identified in animal breeding industries. Studying the effects of 16S rRNA methylase genes on bacterial fitness is meaningful for evaluating the evolution and transmission of this class of genes. In this study, rmtB, one of the most common 16S rRNA methylase genes found in clinical settings, was chosen to probe the fitness impact and phenotypes of 16S rRNA methylase RmtB in order to better understand its persistence of transmitted resistance. 


\section{Materials and methods}

\subsection{Bacterial strains and plasmids}

The strains and plasmids used in the study are listed in Table 1. Bacteria were grown in Luria-Bertani (LB) broth (Oxoid Ltd., Basingstoke, UK; Sinopharm, Shanghai, China) or on LB agar (Oxoid Ltd.; Sinopharm) plates and, when appropriate, media were supplemented with the appropriate antibiotics.

\subsection{Construction of an rmtB in-frame gene deletion mutant, its complemented strain and relevant} recombinant strains

The in-frame $r m t B$ gene of wild-type Escherichia coli 3A11-16 was deleted according to a previously described method with modifications [21,22]. A 1059-bp DNA fragment containing the DNA upstream of $r m t B$ and a 719-bp DNA fragment carrying the DNA downstream of $r m t B$ of $E$. coli strain 3A11-16 were PCR-amplified using an Expand ${ }^{\mathrm{TM}}$ High Fidelity PCR System (TaKaRa, Dalian, China) with two primer pairs: P1/P2 and P3/P4 (Table 2; Fig. 1). Both products were then cloned into the intermediate vector pBluescriptII KS(+) to generate the recombinant pBluescriptII KS(+) $\Delta r m t B$ containing the $r m t B$ gene with an in-frame $r m t B$ deletion. The resulting $r m t B$ in-frame deletion fragment in pBluescriptII KS(+) $\Delta r m t B$ was excised with the restriction enzymes $X b a l$ and $K p n I$ and was inserted into the same restriction enzyme sites in the suicide vector pDMS197, which carries both the $s a c B$ gene (conferring sucrose sensitivity) and tetracycline resistance genes. The recombinant plasmid pDMS197 $\Delta r m t B$ was transformed into E. coli CC118 $\lambda$ pir for replication. Subsequently, the purified recombinant plasmid DNA with the in-frame deletion of $r m t B$ was electroporated into the wild-type strain E. coli 3A11-16. Following electroporation, positive recombinant colonies containing single recombination events were streaked on fresh LB plates containing tetracycline for selection of recombination of plasmid DNA in the bacterial genome. The selected isolates were then grown in nutrient broth containing $10 \%$ sucrose lacking both $\mathrm{NaCl}$ and tetracycline to screen for loss of the integrated $\operatorname{sacB1}$ gene. Colonies sensitive to tetracycline were selected and confirmed by PCR using the primer pair $\Delta r m t B Y Z 1 / \Delta r m t B Y Z 2$ (Table 2; Fig. 1) and their genotypes were examined by PCR using the primer pair P1/P4 (Table 2; Fig. 1) and were sequenced. The deletion mutant was named $E$. coli 3 A11-16 $\Delta r m t B$. Meanwhile, the encoding gene of $r m t B$ was cloned into the pBR322 plasmid for engineering the complemented strain, named $E$. coli $3 A 11-16 \Delta r m t B / p r m t B$, and complementation studies were conducted. 
In addition, in order to study the antibacterial resistance of engineering strains $E$. coli $\mathrm{DH} 5 \alpha$ and $E$. coli BL21(DE3) carrying RmtB, the open-reading frame of $r m t B$ was cloned into multicopy expression vector $\mathrm{pET} 28 \mathrm{a}$ and was transformed into $E$. coli BL21(DE3) resulting in E. coli $\mathrm{BL21}(\mathrm{DE} 3) / \mathrm{pET} 28 \mathrm{a}-\mathrm{rmtB}$, and the recombinant plasmid pBR322-rmtB was transformed into $E$. coli $\mathrm{DH} 5 \alpha$ to generate $E$. coli DH5 $\alpha / \mathrm{pBR} 322-r m t B$.

\subsection{Conjugation experiments}

To determine whether $r m t B$ is located on a conjugative plasmid, conjugation experiments were conducted for E. coli 3A11-16, E. coli 3A11-16 $\Delta r m t B$ and E. coli 3A11-16 $\Delta r m t B / p r m t B$ used as donor bacteria and $E$. coli $\mathrm{C} 600$ as a recipient strain according to a previously described method $[17,23]$. As the plasmids from three types of strains have been confirmed by PCR with primers bla $a_{\text {TEM-1 }} \mathrm{F} /$ bla $_{\text {TEM-1 }} \mathrm{R}$ (Table 2) to carry the same $b / a_{\mathrm{TEM}-1}$ gene, transconjugants were selected on MacConkey agar (Shanghai Zhongke Insect Biology Ltd., Shanghai, China) plates containing both $256 \mathrm{mg} / \mathrm{L}$ ampicillin (Sangon Biotech, Shanghai, China) and 2048 mg/L streptomycin (Sangon Biotech). For the conjugant conjugated from the wild-type strain, transfer of the $r m t B$ and $b / a_{\mathrm{TEM}-1}$ genes was confirmed by PCR with primers $r m t B-\mathrm{U} / r m t B-\mathrm{D}$ and $b / a_{\mathrm{TEM}-1} \mathrm{~F} / \mathrm{b} / a_{\mathrm{TEM}-1} \mathrm{R}$ (Table 2). For the conjugant conjugated from the mutant and complemented strains, transfer of $\Delta r m t B$ and bla TEM-1 $_{1}$ were confirmed by PCR with primers $\Delta r m t B Y Z 1 / \Delta r m t B Y Z 2$ and $b / a_{\mathrm{TEM}-1} \mathrm{~F} / b / a_{\mathrm{TEM}-1} \mathrm{R}$ (Table 2).

\subsection{Antimicrobial susceptibility testing for aminoglycosides}

Minimum inhibitory concentrations (MICs) were determined for the wild-type (E. coli 3A11-16) strain, the $\Delta r m t B$ mutant (E. coli 3A11-16 $\Delta r m t B$ ), the complemented strain (E. coli $3 A 11-16 \Delta r m t B / p r m t B)$ and some relevant recombinant strains using the microdilution susceptibility testing method [24]. Escherichia coli ATCC 25922 was used as a control strain and eight kinds of aminoglycosides were tested, including gentamicin, sisomicin, amikacin, kanamycin, neomycin, apramycin, streptomycin and spectinomycin, of which gentamicin, sisomicin, amikacin and kanamycin are 4,6-disubstituted 2-deoxystreptamines (Table 3).

\subsection{Growth kinetics and growth competition experiments}

Growth kinetics was determined for E. coli 3A11-16, E. coli 3A11-16 $\Delta r m t B$ and E. coli $3 A 11-16 \Delta r m t B / p r m t B$. An inoculum of $10^{7} \mathrm{CFU}$ per strain was grown in $50 \mathrm{~mL}$ of LB broth in the absence of antibiotics. Cultures were incubated for $14 \mathrm{~h}$ at $37^{\circ} \mathrm{C}$ with shaking at $200 \mathrm{rpm}$. The 
optical density at $600 \mathrm{~nm}\left(\mathrm{OD}_{600}\right)$ was measured every 30 min during the first $4 \mathrm{~h}$ and then sequentially every hour after the first $4 \mathrm{~h}$.

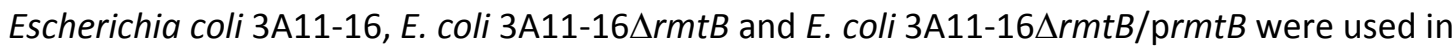
competition experiments as described previously [25]. Bacteria were incubated for $16 \mathrm{~h}$ at $37^{\circ} \mathrm{C} \mathrm{in}$ LB medium and then $10^{6} \mathrm{CFU}$ were mixed in $50 \mathrm{~mL}$ of antibiotic-free LB medium and were grown at $37^{\circ} \mathrm{C}$ with agitation at $100 \mathrm{rpm}$. The cultures were diluted 1:250 into $50 \mathrm{~mL}$ of fresh LB medium every $24 \mathrm{~h}$. Samples were collected every $24 \mathrm{~h}$ for 10 days. Competition experiments were designed such that one competitor carried the aminoglycoside resistance gene $r m t B$ while the other strain did not (i.e. E. coli 3A11-16 vs. E. coli 3A11-16 $\Delta r m t B$ and E. coli 3A11-16 $\Delta r m t B / p r m t B$ vs. E. coli $3 A 11-16 \Delta r m t B)$. Aliquots of bacterial cultures were plated on non-resistance selective LB agar. The proportion of resistant colonies was deduced by replica-plating 100 colonies onto LB agar supplemented with amikacin $(256 \mathrm{mg} / \mathrm{L}$ ) and gentamicin $(256 \mathrm{mg} / \mathrm{L})$.

The competition index $(\mathrm{Cl})$ represents the relative fitness of the bacteria and was calculated as the ratio of the mean CFU in independent competition experiments for the resistant and susceptible strains at a given time point $\left(t_{n}\right)$ divided by the same ratio at initial time $\left(t_{0}\right)$. The selection coefficient (s) was calculated as the slope of the linear regression model: $s=\ln (\mathrm{Cl}) / \ln (d)$, where $d$ is the dilution factor [26]. The selection coefficient (s) estimated the difference between the relative fitness of the two competitors over the entire competition experiment.

\subsection{Biofilm formation and motility assays}

Biofilm assays were performed on microtitre plates as previously described [27] with some modifications. For a quantitative analysis of biofilm production, an overnight culture $\left(\mathrm{OD}_{600}\right.$ of ca. 2.0) was diluted $1: 20$ and was grown in biofilm-inducing medium [27]. Culture medium (100 $\mu \mathrm{L})$ was added to each well of a round-bottomed 96-well microtitre plate (Corning Inc., Corning, NY) and was incubated for $48 \mathrm{~h}$ at $30^{\circ} \mathrm{C}$ without shaking. To remove loosely associated bacteria, the culture medium was removed from the wells, which were rinsed three times with sterile distilled water. Crystal violet (Sinopharm) 1\% solution was added to each well to stain the bacteria. Following incubation at room temperature for $15 \mathrm{~min}$, the wells were rinsed with Milli-Q water and then 95\% ethanol was added to each crystal violet-stained well. The $\mathrm{OD}_{600}$ was measured using a spectrophotometer (BioTek, Winooski, VT). Six replicates were used in each experiment and each experiment was performed three times. 
Motility assays were performed on semisolid agar plates as described previously [28] with some modifications. Briefly, $50 \mathrm{~mL}$ of bacterial stock from overnight cultures was re-inoculated into $5 \mathrm{~mL}$ of sterile LB broth and was incubated at $37^{\circ} \mathrm{C}$ with aeration in a bacterial shaker at $178 \mathrm{rpm}$ until an $\mathrm{OD}_{600}$ of ca. 1.0 was achieved, indicating that the culture had entered logarithmic phase. Then, $1 \mu \mathrm{L}$ of the culture samples from the E. coli 3A11-16 parent strain, the mutant $E$. coli 3A11-16 $\Delta r m t B$ strain and the complemented $E$. coli $3 A 11-16 \Delta r m t B / p r m t B$ strain were seeded onto $0.3 \%$ semisolid tryptone agar plates (Oxoid Ltd., Sinopharm). Motility was observed and valued following incubation for $18 \mathrm{~h}$ at $37^{\circ} \mathrm{C}$ by measuring the diameter of radial diffusion around the primary inoculum.

\section{Results and discussion}

\subsection{Construction of the $\triangle r m t B$ mutant and the complemented strain}

Upstream and downstream homologous fragments of $r m t B$ covering lengths of $1059 \mathrm{bp}$ and $719 \mathrm{bp}$, respectively, were successfully amplified by PCR, yielding products with the expected size. After confirming the DNA sequence of the PCR product, $30 \mathrm{bp}$ (residues 25-54) of the $r m t B$ gene in E. coli 3A11-16 was successfully deleted using the double selection strategy of allelic exchange mutagenesis with suicide vector pDMS197-based recombination. Generally the $r m t B$ resistance gene is located on conjugative or non-conjugative plasmids $[17,29,30]$ and, in this study, we confirmed that the $r m t B$ gene of the $E$. coli strain used was located on a conjugative plasmid using conjugation experiments. Edwards et al. [22] demonstrated that genes on the chromosome and on plasmids could be modified through suicide vector-mediated recombination. In the current study, we demonstrated that the gene on the conjugative plasmid could be modified using suicide vector-based recombination, and homologous recombination could occur on both the chromosome and the plasmid in the prokaryotic cells [22].

For the complemented strain, after tetracycline-resistant transformants were selected and confirmed with proof primers $\Delta r m t B Y Z 1 / \Delta r m t B Y Z 2$, the recombinant plasmid pBR322 carrying the $r m t B$ gene was confirmed by both PCR analysis using primers $\mathrm{pBR} 322-\mathrm{F} / \mathrm{pBR} 322-\mathrm{R}$ and $r m t B-\mathrm{U} /$ $r m t B-D$ and DNA sequencing, the complemented strain $E$. coli 3A11-16 $\Delta r m t B / p r m t B$ was constructed successfully. 


\subsection{RmtB-harbouring strains show high-level resistance to 4,6-disubstituted 2-deoxystreptamines}

As shown in Table 3, the MICs of the 4,6-disubstituted 2-deoxystreptamines (i.e. gentamicin, sisomicin, amikacin and kanamycin ) for the $\Delta r m t B$ mutant were significantly reduced to the same level as the control strain E. coli ATCC 25922. However, there was no significant difference between the $r m t B$ wild-type and the $\Delta r m t B$ mutant in the MICs of non-4,6-disubstituted 2-deoxystreptamines. As expected, there was a marked difference observed in the MICs of the 4,6-disubstituted 2-deoxystreptamines between the $\Delta r m t B$ mutant and the complemented strain. The MICs of the 4,6-disubstituted 2-deoxystreptamines of the complemented strain were restored to wild-type levels. However, differences in the MICs of non-4,6-disubstituted 2-deoxystreptamines between the wild-type and the $\Delta r m t B$ mutant were not significant, and similar results were observed both in E. coli DH5 $\alpha$ carrying pBR322 and E. coli BL21(DE3) carrying pET28a whether or not they express the methylase gene $r m t B$.

Following deletion of the target gene in the 16S rRNA methylase-producing strain and phenotypic analysis for the clinical prototype strain, its isogenic mutant and the complemented strain, the resistance mechanism could be further elucidated. As already known, methylation of the N1 position of A1408 in 16S rRNA of bacteria confers resistance to 4,6-disubstituted 2-deoxystreptamines (kanamycin and gentamicin groups), 4,5-disubstituted 2-deoxystreptamines (neomycin group) and apramycin, a monosubstituted 2-deoxystreptamines, whereas methylation of N7 G1405 confers resistance to only 4,6-disubstituted 2-deoxystreptamines [4,11,30]. 16S rRNA methylase RmtB may act by directly blocking aminoglycoside binding to the target site, i.e. N7 G1405, in the A site of $16 \mathrm{~S}$ rRNA, and this would confer bacterial resistance to various aminoglycosides belonging to the 4,6-disubstituted 2-deoxystreptamines. The susceptibility assay results show a significant difference between the wild-type and mutant strains against 4,6-disubstituted 2-deoxystreptamines. The susceptibility of the $\Delta r m t B$ mutant to 4,6-disubstituted 2-deoxystreptamines showed that deleting the amino acid residues 9-18 (corresponding to base pairs 25-54) of RmtB likely disrupts the whole protein structure. Schmitt et al. [31] demonstrated that the N-terminal domain specific to the aminoglycoside resistance family (Agr) of methyltransferases is required for enzymatic activity and amino acid residues 10-19 of RmtB are strictly conserved, as observed by analysis of the three-dimensional structure of RmtB. Based on a mutation of 10 amino acid residues from positions 9-18 of RmtB, leading to the $\Delta r m t B$ mutant's sensitivity to 4,6-disubstituted 2-deoxystreptamines, we reason that amino acid residues 9-18 of RmtB may play a critical role in methylating the $A$ site of 16S rRNA by S-adenosylmethionine, which needs to be confirmed. 


\subsection{The growth rate is relatively slower in rmtB-harbouring strains}

From the bacterial growth curves (Fig. 2) it can be observed that the growth rates both of $E$. coli 3A11-16 and E. coli 3A11-16 $\Delta r m t B / p r m t B$ were slightly lower than that of the $\Delta r m t B$ mutant after the exponential phase. The fitness cost associated with most resistance mechanisms is an important biological parameter that influences the emergence and dissemination of antimicrobial resistance and its persistence in the absence of selective pressure [32]. Considering that the growth rate of $E$. coli 3A11-16 $\Delta r m t B / p r m t B$ was significantly lower compared with $E$. coli $3 A 11-16 \Delta r m t B$, whereas the growth rate of $E$. coli 3A11-16 was only slightly lower (Fig. 2), we suggest that the 16S rRNA methylase RmtB, which confers methylation at N7 G1405 of the A site of 16S rRNA, relates to a small disadvantage that causes a slightly decreased growth after the exponential phase, especially in the condition when $r m t B$ is multicopy-expressed. Benítez-Páez et al. reported that aminoglycoside methylase RsmG, methylating $\mathrm{m}^{7} \mathrm{G} 527$ in $16 \mathrm{~S}$ rRNA, stimulates ppGpp accumulation and downregulates rRNA synthesis when cells were grown in nutrient-limitation conditions that cause growth arrest or were entered into the stationary phase [33]. The current research results perhaps suggest that a similar mechanism exists in 165 rRNA methylase RmtB in E. coli.

\subsection{The competitive capacity of the rmtB-harbouring strain is reduced}

In vitro competition experiments were performed between three strains that differed by the presence or absence of the $16 \mathrm{~S}$ rRNA methylase RmtB gene in its active or inactive form. An obvious competitive disadvantage was observed in $r m t B$ wild-type compared with the $\Delta r m t B$ mutant, with a loss of $3.25 \%$ per generation (Fig. 3A). Moreover, a major competitive disadvantage was observed in cells transformed with the recombinant plasmid pBR322-rmtB expressing RmtB compared with the mutant, with a loss of $20.89 \%$ per generation (Fig. 3B). A marked competitive disadvantage was observed for the wild-type and cells with multicopy-expressing RmtB, thus confirming that 16S rRNA methylase RmtB, leading to N7 G1405 methylation, is responsible for exacting a fitness cost to the cells.

The slight biological cost associated with resistance could lead to the selection of compensatory mutations that restore the fitness of the host [34]. However, in contrast to RmtC, the results of growth rate and growth competition experiments showed that the 16S rRNA methylase RmtB leads to a fitness burden to the host bacteria, as observed for most other antimicrobial resistance enzymes. 


\subsection{Acquisition of $16 \mathrm{~S}$ rRNA methylase RmtB has no impact on biofilm formation, motility or}

conjugation of the host bacterium

Although the amount of biofilm formation for the mutant was found to be consistently higher than that of the wild-type strain, no significant difference was found in biofilm formation among the three types of strains (Fig. 4A). Biofilm formation and its contribution to resistance to antimicrobial agents may be a major cause of many persistent and chronic infections. The environment of the bacterial biofilm shows increased tolerance to antibiotics and disinfectant chemicals [35], since the dense extracellular matrix and the outer layer of cells protect the interior of the bacterial community. In some cases, antibiotic resistance could be increased 1000-fold owing to the existence of biofilms [36]. Høiby et al. reported that conventional resistance mechanisms, such as chromosomal $\beta$-lactamase, upregulated efflux pumps and mutations in antibiotic target molecules in bacteria, also enhance biofilm survival [35]. To understand how bacteria adapt to acquisition of the $r m t B$ gene, the biofilm formation experiment and motility tests were performed. Contrary to the report of $\mathrm{H} \varnothing \mathrm{iby}$ et al. mentioned above [35], we found that expression of 16S rRNA methylase RmtB did not enhance biofilm formation and instead led to a slight decline in biofilm formation. Moreover, no significant discrepancy was found in the motility of the three strains (Fig. 4B).

In addition, transconjugants were easily obtained for each of the three types of strains in conjugation experiments. The resistance plasmid of the $\Delta r m t B$ mutant can still be transferred into the recipient strain, confirming that the $16 \mathrm{~S}$ rRNA methylase $r m t B$ gene does not influence the function of the conjugation apparatus (or tra genes) of the conjugative resistance plasmid.

The effects of aminoglycoside methylases on bacterial fitness have been reported as being highly variable. Punekar et al. stated that knockout of $r / m J$ (yhiR), encoding methylase RImJ (YhiR) methylating $\mathrm{m}^{6} \mathrm{~A} 2030$ in $23 \mathrm{~S}$ rRNA, does not affect the growth rate $[14,15]$ but lowers the competitive fitness at long-term growth in stationary phase $[15,16]$ and provides a small growth advantage under anaerobic conditions [14,15]. However, no fitness cost coupled with the methylation pattern at N7 G1405 of the 16S rRNA of acquired 16S rRNA methylase (or Arm/Rmt methylase) RmtC was observed, which suggests that evolution of the ribosome ultimately led to the selection and maintenance of resistance even in the absence of selective pressure [25]. Different from that of the 16S rRNA methylase RmtC, acquisition of 16S rRNA methylase RmtB located on a conjugation plasmid decreases the host bacterial fitness by reducing the growth rate slightly and decreasing the competitive power of E. coli. Therefore, the fitness costs of the acquired 16S rRNA methylase RmtB do not explain the phenomenon of the increasing prevalence of the acquisition of 
the $16 \mathrm{~S}$ rRNA methylase gene $r m t B$ in clinical settings, and more research work should be carried out to illustrate the mechanism in the future.

Moreover, exploration of the difference between the wild-type $r m t B$ strain and its isogenic $\Delta r m t B$ mutant may facilitate the potential exploitation of effective inhibitors of $16 \mathrm{~S}$ rRNA methylase, which will be of vital importance in the near future.

Acknowledgments: The authors are grateful to Prof. Jianhua Liu (South China Agricultural University, Guangzhou, China) for providing recipient strain E. coli C600. The authors also thank Dieter M. Schifferli (Veterinary Microbiology Laboratory, University of Pennsylvania, Philadelphia, PA) for providing pDMS197 and E. coli CC118 $\lambda$ pir.

Funding: This study was supported by grants from the Chinese National Science Foundation [grant nos. 30571374, 30771603, 31072136 and 31270171] and the Genetically Modified Organisms Technology Major Project of China [2014ZX08006-001B], a project founded by the Jiangsu Overseas Research \& Training Program for University Prominent Young and Middle-aged Teachers and Presidents and the China Postdoctoral Science Foundation [2013M541740], and the Jiangsu Innovation Research Program for university postgraduates [KYLX15_1382].

Competing interests: None declared.

Ethical approval: Not required. 


\section{References}

[1] Magnet S, Blanchard JS. Molecular insights into aminoglycoside action and resistance. Chem Rev 2005;105:477-98.

[2] Cundliffe E. How antibiotic-producing organisms avoid suicide. Annu Rev Microbiol 1989;43:207-33.

[3] Yokoyama K, Doi Y, Yamane K, Kurokawa H, Shibata N, Shibayama K, et al. Acquisition of $16 \mathrm{~S}$ rRNA methylase gene in Pseudomonas aeruginosa. Lancet 2003;362:1888-93.

[4] Doi Y, Arakawa Y. 16 S ribosomal RNA methylation: emerging resistance mechanism against aminoglycosides. Clin Infect Dis 2007;45:88-94.

[5] Doi Y, Garcia DD, Adams J, Paterson DL. Coproduction of novel $16 S$ rRNA methylase RmtD and metallo- $\beta$-lactamase SPM-1 in a panresistant Pseudomonas aeruginosa isolate from Brazil. Antimicrob Agents Chemother 2007;51:852-6.

[6] Tijet N, Andres P, Chung C, Lucero C, WHONET Argentina Group; Low DE, et al. rmtD2, a new allele of a 16S rRNA methylase gene, has been present in Enterobacteriaceae isolates from Argentina for more than a decade. Antimicrob Agents Chemother 2011;55:904-9.

[7] Davis MA, Baker KN, Orfe LH, Shah DH, Besser TE, Call DR. Discovery of a gene conferring multiple-aminoglycoside resistance in Escherichia coli. Antimicrob Agents Chemother 2010;54:2666-9.

[8] Galimand M, Courvalin P, Lambert T. RmtF, a new member of the aminoglycoside resistance 16S rRNA N7 G1405 methyltransferase family. Antimicrob Agents Chemother 2012;56:3960-2.

[9] Bueno MFC, Francisco GR, O'Hara JA, de Oliveira Garcia D, Doi Y. Coproduction of 16S rRNA methyltransferase RmtD or RmtG with KPC-2 and CTX-M group extended-spectrum $\beta$-lactamases in Klebsiella pneumoniae. Antimicrob Agents Chemother 2013;57:2397-400.

[10]O'Hara JA, McGann P, Snesrud EC, Clifford RJ, Waterman PE, Lesho EP, et al. Novel 16S rRNA methyltransferase RmtH produced by Klebsiella pneumoniae associated with war-related trauma. Antimicrob Agents Chemother 2013;57:2413-6.

[11]Wachino J, Shibayama K, Kurokawa H, Kimura K, Yamane K, Suzuki S, et al. Novel plasmid-mediated 16S rRNA m1A1408 methyltransferase, NpmA, found in a clinically isolated Escherichia coli strain resistant to structurally diverse aminoglycosides. Antimicrob Agents Chemother 2007;51:4401-9. 
[12]Andersson DI. The biological cost of mutational antibiotic resistance: any practical conclusions? Curr Opin Microbiol 2006;9:461-5.

[13]Vogwill T, MacLean RC. The genetic basis of the fitness costs of antimicrobial resistance: a meta-analysis approach. Evol Appl 2015;8:284-95.

[14]Golovina AY, Dzama MM, Osterman IA, Sergiev PV, Serebryakova MV, Bogdanov AA, et al. The last rRNA methyltransferase of $E$. coli revealed: the $y$ hiR gene encodes adenine-N6 methyltransferase specific for modification of A2030 of 23 S ribosomal RNA. RNA 2012;18:1725-34.

[15]Punekar AS, Liljeruhm J, Shepherd TR, Forster AC, Selmer M. Structural and functional insights into the molecular mechanism of rRNA m6A methyltransferase RImJ. Nucleic Acids Res 2013;41:9537-48.

[16]Palchevskiy V, Finkel SE. Escherichia coli competence gene homologs are essential for competitive fitness and the use of DNA as a nutrient. J Bacteriol 2006;188:3902-10.

[17]Chen L, Chen ZL, Liu JH, Zeng ZL, Ma JY, Jiang HX. Emergence of RmtB methylase-producing Escherichia coli and Enterobacter cloacae isolates from pigs in China. J Antimicrob Chemother 2007;59:880-5.

[18]Yu FY, Yao D, Pan JY, Chen C, Qin ZQ, Parsons C, et al. High prevalence of plasmid-mediated 16S rRNA methylase gene $r m t B$ among Escherichia coli clinical isolates from a Chinese teaching hospital. BMC Infect Dis 2010;10:184.

[19]Yamane K, Wachino J, Doi Y, Kurokawa H, Arakawa Y. Global spread of multiple aminoglycoside resistance genes. Emerg Infect Dis 2005;11:951-3.

[20]Galani I, Souli M, Panagea T, Poulakou G, Kanellakopoulou K, Giamarellou H. Prevalence of 16S rRNA methylase genes in Enterobacteriaceae isolates from a Greek university hospital. Clin Microbiol Infect 2012;18:E52-4.

[21]Zhu C, Meng X, Duan X, Tao Z, Gong J, Hou H, et al. SEF14 fimbriae from Salmonella enteritidis play a role in pathogenitic to cell model in vitro and host in vivo. Microb Pathog 2013;64:18-22.

[22]Edwards RA, Keller LH, Schifferli DM. Improved allelic exchange vectors and their use to analyze 987P fimbria gene expression. Gene 1998;207:149-57. 
[23]Hou J, Huang $X$, Deng $Y$, He L, Yang T, Zeng Z, et al. Dissemination of fosfomycin resistance gene fosA 3 with CTX-M $\beta$-lactamase genes and $r m t B$ carried on IncFIl plasmids among Escherichia coli isolates from pets in China. Antimicrob Agents Chemother 2012;56:2135-8.

[24]Clinical and Laboratory Standards Institute. Performance standards for antimicrobial susceptibility testing; twenty-second informational supplement. Document M100-S22. Wayne, PA: CLSI; 2012.

[25]Gutierrez B, Escudero JA, San Millan A, Hidalgo L, Carrilero L, Ovejero CM, et al. Fitness cost and interference of Arm/Rmt aminoglycoside resistance with the RsmF housekeeping methyltransferases. Antimicrob Agents Chemother 2012;56:2335-41.

[26]San Millan A, Garcia-Cobos S, Escudero JA, Hidalgo L, Gutierrez B, Carrilero L, et al. Haemophilus influenzae clinical isolates with plasmid pB1000 bearing $b / a_{\mathrm{ROB}-1}$ : fitness cost and interspecies dissemination. Antimicrob Agents Chemother 2010;54:1506-11.

[27] Hossain MM, Tsuyumu S. Flagella-mediated motility is required for biofilm formation by Erwinia carotovora subsp. carotovora. J Gen Plant Pathol 2006;72:34-9.

[28]Lane MC, Simms AN, Mobley HLT. Complex interplay between type 1 fimbrial expression and flagellum-mediated motility of uropathogenic Escherichia coli. J Bacteriol 2007;189:5523-33.

[29]Bogaerts P, Galimand M, Bauraing C, Deplano A, Vanhoof R, De Mendonca R, et al. Emergence of ArmA and RmtB aminoglycoside resistance 16S rRNA methylases in Belgium. J Antimicrob Chemother 2007;59:459-64.

[30]Doi Y, Yokoyama K, Yamane K, Wachino J, Shibata N, Yagi T, et al. Plasmid-mediated $16 \mathrm{~S}$ rRNA methylase in Serratia marcescens conferring high-level resistance to aminoglycosides. Antimicrob Agents Chemother 2004;48:491-6.

[31]Schmitt E, Galimand M, Panvert M, Courvalin P, Mechulam Y. Structural bases for 16 S rRNA methylation catalyzed by ArmA and RmtB methyltransferases. J Mol Biol 2009;388:570-82.

[32]Andersson DI, Hughes D. Antibiotic resistance and its cost: is it possible to reverse resistance? Nat Rev Microbiol 2010;8:260-71.

[33]Benítez-Páez A, Villarroya M, Armengod M-E. Regulation of expression and catalytic activity of Escherichia coli RsmG methyltransferase. RNA 2012;18:795-806.

[34]Foucault ML, Courvalin P, Grillot-Courvalin C. Fitness cost of VanA-type vancomycin resistance in methicillin-resistant Staphylococcus aureus. Antimicrob Agents Chemother 2009;53:2354-9. 
[35]Høiby N, Bjarnsholt T, Givskov M, Molin S, Ciofu O. Antibiotic resistance of bacterial biofilms. Int J Antimicrob Agents 2010;35:322-32.

[36]Stewart PS, Costerton JW. Antibiotic resistance of bacteria in biofilms. Lancet 2001;358:135-8. 
Fig. 1. Schematic diagrams for construction of deletion mutations. (A) Schematic diagram for the position of primers (grey boxes represent the gene open reading frame). (B) Schematic diagram for rmtB deletion; 30 base pairs (residues 25-54) of the rmtB gene were deleted and a 57-bp exogenous gene was inserted into the original place [hatched part represents exogenous fragment from the pBluescriptII KS(+)].

Fig. 2. Growth kinetics of three Escherichia coli strains: wild-type E. coli 3A11-16; rmtB deletion mutant (E. coli 3A11-16 $\Delta r m t B$ ); and the complemented strain (E. coli 3A11-16 $\Delta r m t B / p r m t B)$. OD $_{600}$, optical density at $600 \mathrm{~nm}$. Data are the mean \pm standard error of the mean.

Fig. 3. Fitness cost of RmtB in different backgrounds. Growth competition curves between three types of strains. (A) Escherichia coli 3A11-16 wild-type and E. coli 3A11-16 $\Delta r m t B$ deletion mutant. (B) E. coli 3A11-16 $\Delta r m t B$ and complemented strain E. coli 3A11-16 $\Delta r m t B / p r m t B$. Competition curves are depicted as the mean of three independent experiments. Data are the mean \pm standard error of the mean. An obvious competitive disadvantage with a loss of $3.25 \%$ per generation was observed for $r m t B$ wild-type compared with the $\Delta r m t B$ mutant; and a major competitive disadvantage with a loss of $20.89 \%$ per generation was observed in cells transformed with the recombinant plasmid pBR322-rmtB expressing RmtB compared with the mutant. $\mathrm{Cl}$, competition index.

Fig. 4. Biofilm formation and motility of the $16 \mathrm{~S}$ rRNA methylase RmtB-positive strains and their negative counterpart. (A) Quantification of biofilm formation of Escherichia coli 3A11-16 wild-type (WT), the E. coli 3A11-16 $\Delta r m t B$ deletion mutant and the E. coli 3A11-16 $\Delta r m t B / p r m t B$ complemented strain. Surface-adhered biofilm was quantified by measuring the optical density at $600 \mathrm{~nm}\left(\mathrm{OD}_{600}\right)$ of ethanol-solubilised crystal violet after biofilm staining from three independent experiments. (B) Motility assay of E. coli 3A11-16 WT, the E. coli 3A11-16 $\Delta r m t B$ deletion mutant and the $E$. coli $3 A 11-16 \Delta r m t B / p r m t B$ complemented strain. Motility was visualised as diameter of radial diffusion around the primary inoculum on semisolid agar plates after $18 \mathrm{~h}$ at $37^{\circ} \mathrm{C}$. Data are the mean \pm standard error of the mean. 


\section{Table 1}

Strains and plasmids used in this work

\begin{tabular}{|c|c|c|}
\hline Strain or plasmid & Genotype & Resource \\
\hline \multicolumn{3}{|l|}{ Strains } \\
\hline E. coli $3 \mathrm{~A} 11-16$ & $\begin{array}{l}\text { rmtB-positive wild-type Escherichia colifrom } \\
\text { chicken }\end{array}$ & $\begin{array}{l}\text { Clinical } \\
\text { collection }\end{array}$ \\
\hline $\begin{array}{l}\text { E. coli ATCC } \\
25922\end{array}$ & Drug-sensitive control strain & ATCC stock \\
\hline E. coli $\mathrm{DH} 5 \alpha$ & $\begin{array}{c}\mathrm{F}^{-}, \varphi 80 \mathrm{~d} / \text { lacZ } \Delta \mathrm{M} 15, \Delta(\text { lacZYA-argF)U169, } \\
\text { deoR, recA1, endA1, hsdR17(rk-, mk+), } \\
\text { phoA, supE44, } \lambda-, \text { thi-1, gyrA96, relA1 }\end{array}$ & Takara Ltd. \\
\hline E. coli CC118 גpir & $\begin{array}{l}\operatorname{araD139} \Delta(\text { araleu }) 7697 \Delta l a c X 74 \Delta \text { phoA20 } \\
\text { galKthirpsErpoBargE(Am) recA1 (Apir) }\end{array}$ & [22] \\
\hline E. coli $\mathrm{C} 600$ & $\begin{array}{l}\text { Streptomycin-resistant }(\mathrm{MIC}>2000 \mathrm{mg} / \mathrm{L}) \text { as } \\
\text { recipient strain }\end{array}$ & [23] \\
\hline \multicolumn{3}{|l|}{ Plasmids } \\
\hline pDMS197 & oriT, oriV, sacB, $T c^{r}$ & [22] \\
\hline pDMS197- $\Delta r m t B$ & pDMS197 carrying in-frame $\Delta r m t B$ gene, $T c^{r}$ & This study \\
\hline $\begin{array}{l}\text { pBluescriptll } \\
\mathrm{KS}(+)\end{array}$ & Intermediate vector, $\mathrm{Amp}^{\mathrm{r}}$ & $\begin{array}{l}\text { Stratagene, } \\
\text { laboratory } \\
\text { stock }\end{array}$ \\
\hline $\begin{array}{l}\text { pBluescriptll } \\
\mathrm{KS}(+) \Delta r m t B\end{array}$ & $\begin{array}{l}\text { pBluescriptll KS(+) carrying in-frame } \Delta r m t B \\
\text { gene }\end{array}$ & This study \\
\hline pBR322 & Expression vector, $\mathrm{Tc}^{r}, \mathrm{Amp}^{\mathrm{r}}$ & Takara Ltd. \\
\hline pBR322-rmtB & Intact $r m t B$ ligated into pBR322, $\mathrm{Tc}^{r}$ & This study \\
\hline pET28a & Bacterial expression vector, $\mathrm{Kan}^{\mathrm{r}}$ & Takara Ltd. \\
\hline pET28a-rmtB & Intact $r m t B$ ligated into $\mathrm{pET} 28 \mathrm{a}, \mathrm{Kan}^{\mathrm{r}}$ & This study \\
\hline
\end{tabular}


MIC, minimum inhibitory concentration; $\mathrm{Tc}^{r}$, tetracycline-resistant; $\mathrm{Amp}^{r}$, ampicillin-resistant; Kan', kanamycin-resistant. 


\section{Table 2}

Primers used in this study

\begin{tabular}{|c|c|c|}
\hline \multicolumn{3}{|c|}{$\begin{array}{l}\text { Table } 2 \\
\text { Primers used in this study }\end{array}$} \\
\hline Primer name & Sequence & Description \\
\hline $\mathrm{P} 1$ & GCTCTAGACATTTTCGTGTCGCCCTTATT (Xbal) & For amplifying homologous sequence; amplified size 1059 bp \\
\hline $\mathrm{P} 2$ & CGCGGATCCGGTGAGGGCATCGTTGA (BamHI) & \\
\hline P3 & CCGTCGAGCTTTGCCCGGATACC (Xhol) & For amplifying homologous sequence; amplified size 719 bp \\
\hline P4 & GGGGTACCTTATCCATTCTTTTTTATCAAGT (Kpnl) & \\
\hline$r m t B-U$ & AAAAGTACTATGAACATCAACGATGCCCT (Scal) & Construction of pBR322-rmtB, amplified size 774 bp \\
\hline$r m t B-\mathrm{D}$ & AAAAGTACTTTATCCATTCTTTTTTATCA (Scal) & \\
\hline$\Delta r m t B Y Z 1$ & GGCTGCAGGAATTCGATATCAAG & $\Delta r m t B$ mutant confirmation; amplified size 237 bp \\
\hline$\Delta r m t B Y Z 2$ & CATGCAGCGACAATGCCTT & \\
\hline blaTEM-1 $\mathrm{F}$ & AACATTTTCGTGTCGCCCTT & blaTEM-1 gene confirmation; amplified size 226 bp \\
\hline bla TEM-1 $R$ & AATACCGCACCACATAGCAG & \\
\hline pBR322-F & ACGCAGTCAGGCACCGTGTAT & pBR322 plasmid confirmation; amplified size 1247 bp \\
\hline pBR322-R & GTGGTGAATCCGTTAGCGAGG & \\
\hline
\end{tabular}

Note: Nucleotides underlined represent the restriction sites labelled in parentheses. 


\section{Table 3}

Antimicrobial susceptibilities of the $r m t B$ wild-type strain $E$. coli $3 A 11-16$, the $\Delta r m t B$ deletion mutant $E$. coli $3 A 11-16 \Delta r m t B$, the complemented strain E. coli $3 \mathrm{~A} 11-16 \Delta r m t B / p r m t B$, and some relevant recombinant strains

\begin{tabular}{lllllllll}
\hline \multirow{2}{*}{ Strain } & \multicolumn{1}{l}{ MIC $(\mathrm{mg} / \mathrm{L})$} \\
\cline { 2 - 8 } & GEN & SISO & KAN & AMK & NEO & APR & STR & SPE \\
\hline E. coli 3A11-16 (wild-type $r m t B)$ & 1024 & 1024 & $>1024$ & $>1024$ & 4 & 4 & 8 & 64 \\
E. coli 3A11-16 $\Delta r m t B$ & 1 & 1 & 2 & 1 & 4 & 2 & 4 & 128 \\
E. coli 3A11-16 $\Delta r m t B / p r m t B$ & $>1024$ & $>1024$ & $>1024$ & $>1024$ & 4 & 2 & 4 & 32 \\
E. coli DH5 $\alpha /$ pBR322 & 1 & 1 & 2 & 1 & 2 & 1 & 1 & 32 \\
E. coli DH5 $\alpha / p B R 322-r m t B$ & 1024 & 256 & 1024 & 1024 & 1 & 1 & 0.5 & 32 \\
E. coli BL21(DE3)/pET28a & 1 & 1 & 256 & 2 & 32 & 2 & 4 & 32 \\
E. coli BL21(DE3)/pET28a-rmtB & 512 & 512 & $>1024$ & $>1024$ & 64 & 4 & 8 & 128 \\
E. coli ATCC 25922 & 1 & 1 & 2 & 1 & 4 & 2 & 4 & 64 \\
\hline
\end{tabular}

MIC, minimum inhibitory concentration; GEN, gentamicin; SISO, sisomicin; KAN, kanamycin; AMK, amikacin; NEO, neomycin; APR, apramycin; STR, streptomycin; SPE, spectinomycin. 

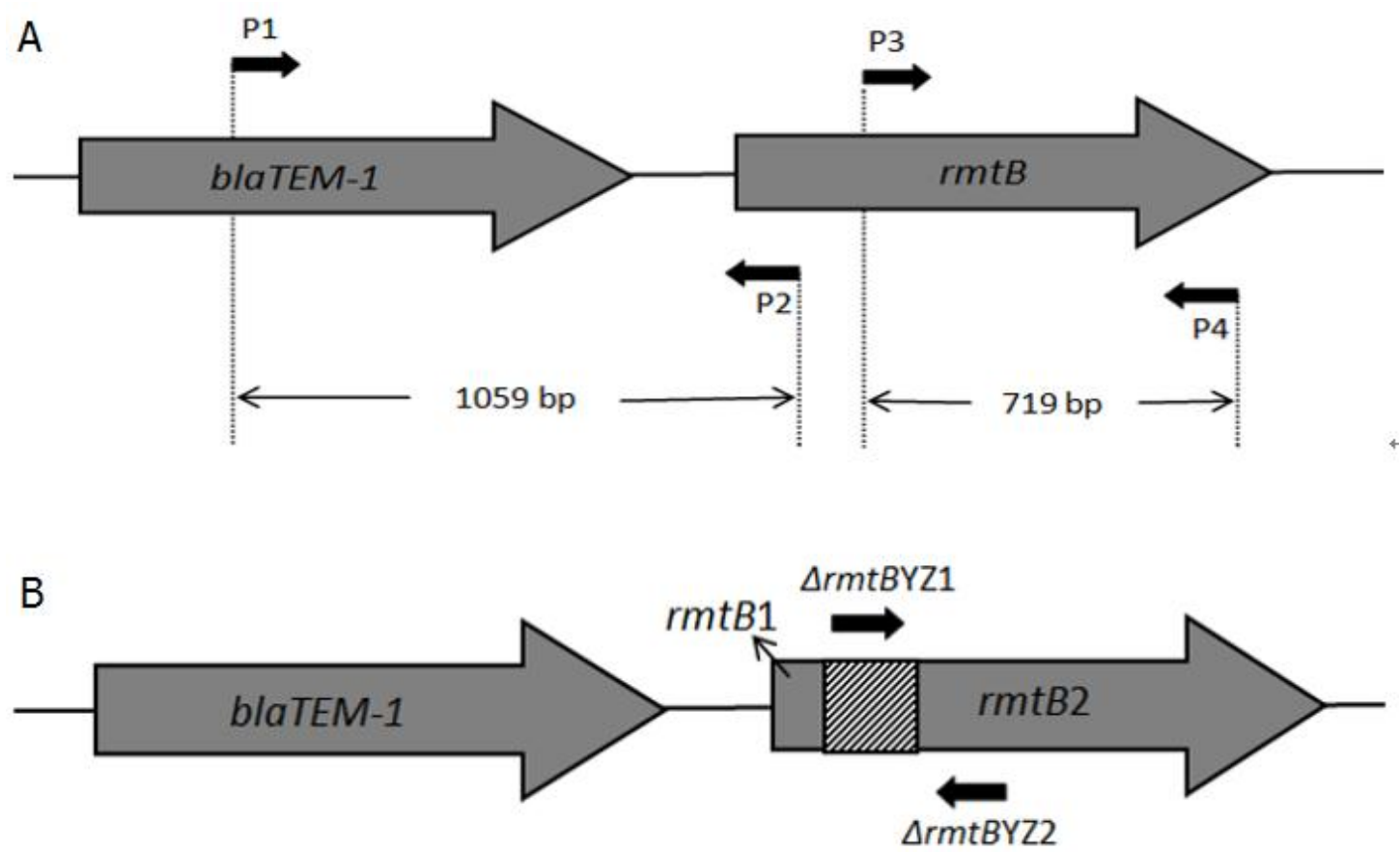


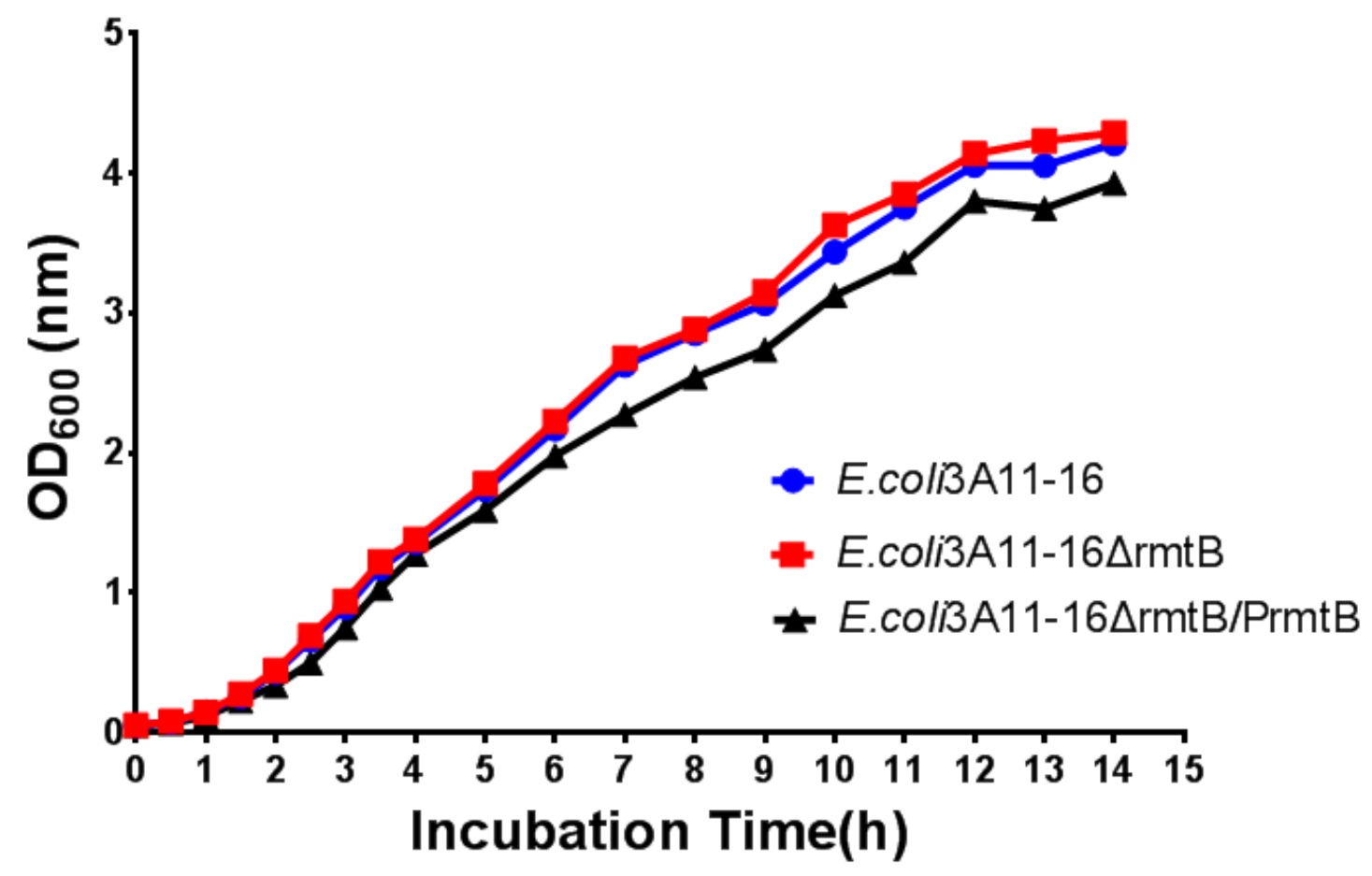



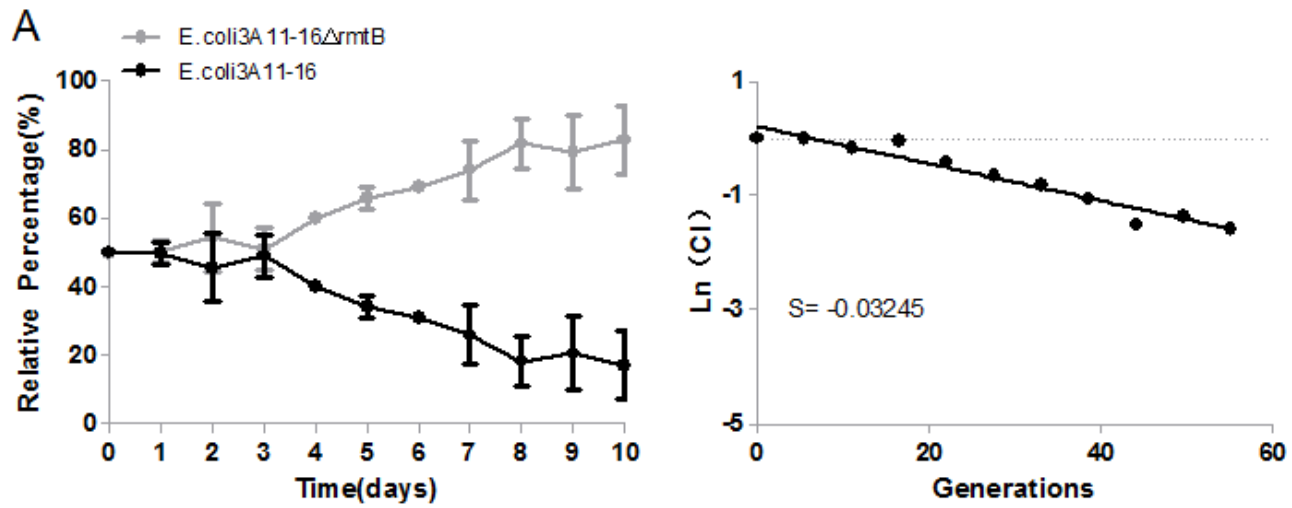

B $\rightarrow$ E.coli3A 11-16 $\Delta$ mtB
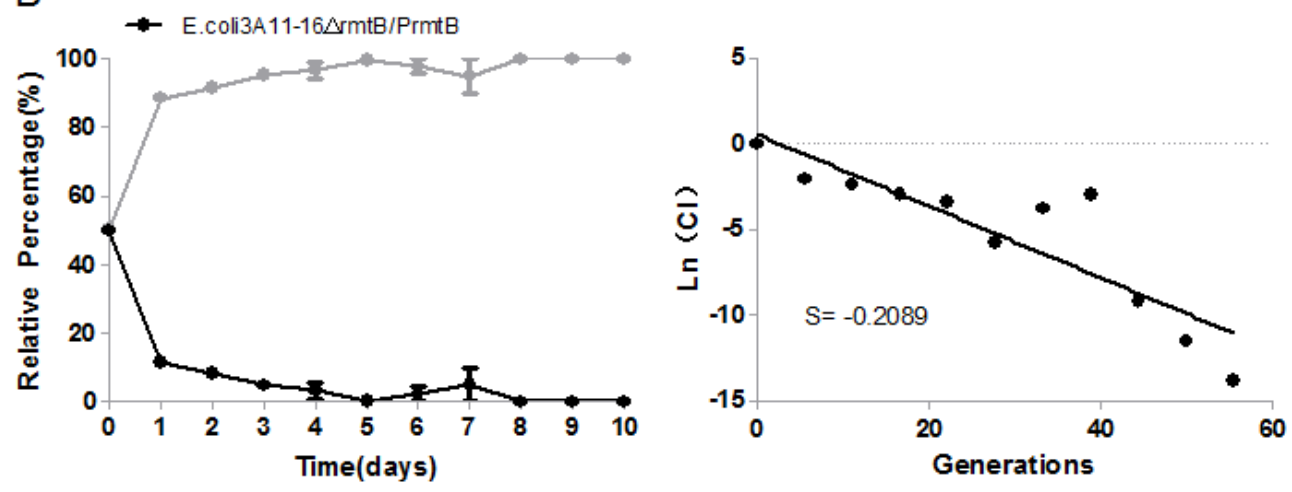
A

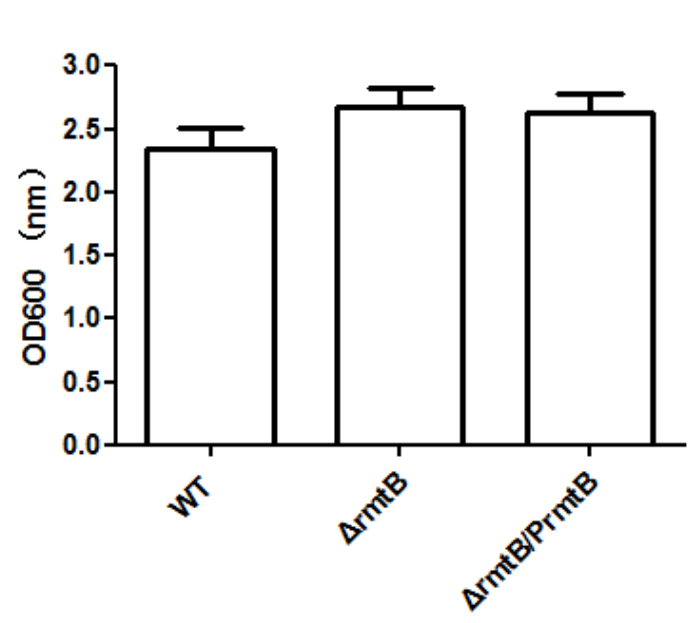

B

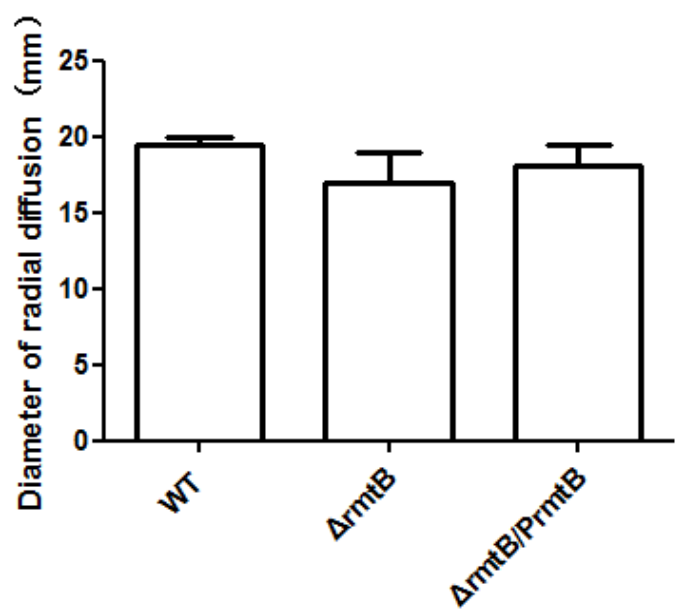

